\title{
The Complexity of Finding Reset Words in Finite Automata
}

\author{
Jörg Olschewski ${ }^{1,2, \star}$ and Michael Ummels ${ }^{2,3, \star \star}$ \\ 1 Lehrstuhl Informatik 7, RWTH Aachen University, Germany \\ olschewski@automata.rwth-aachen.de \\ 2 LSV, CNRS \& ENS Cachan, France \\ 3 Mathematische Grundlagen der Informatik, \\ RWTH Aachen University, Germany \\ ummels@logic.rwth-aachen.de
}

\begin{abstract}
We study several problems related to finding reset words in deterministic finite automata. In particular, we establish that the problem of deciding whether a shortest reset word has length $k$ is complete for the complexity class DP. This result answers a question posed by Volkov. For the search problems of finding a shortest reset word and the length of a shortest reset word, we establish membership in the complexity classes $\mathrm{FP}^{\mathrm{NP}}$ and $\mathrm{FP}^{\mathrm{NP}[\log ]}$, respectively. Moreover, we show that both these problems are hard for $\mathrm{FP}^{\mathrm{NP}[\mathrm{log}]}$. Finally, we observe that computing a reset word of a given length is FNP-complete.
\end{abstract}

\section{Introduction}

A synchronising automaton is a deterministic finite automaton that can be reset to a single state by reading a suitable word. More precisely, there needs to exist a word $w$ such that, no matter at which state of the automaton we start, $w$ takes the automaton to the same state $q$; we call any such word $w$ a reset word or a synchronising word. Although it is easy to decide whether a given automaton is synchronising and to compute a reset word, finding a shortest reset word seems to be a hard problem.

The motivation to study reset words does not only come from automata theory: There are applications in the fields of many-valued logics, biocomputing, set theory, and many more [12]. A purely mathematical viewpoint can be obtained by identifying letters with their associated transition functions, which act on a finite set. The task is then to find a composition of these functions such that the resulting function is constant.

The theory of synchronising automata has been established in the 1960s and is still actively developed. The famous Černý Conjecture was formulated in 1971 3. The conjecture claims that every synchronising automaton with $n$ states has a reset word of length $(n-1)^{2}$. As of now, the conjecture has neither been

* supported by the ESF project GASICS.

${ }^{\star \star}$ supported by the French project DOTS (ANR-06-SETI-003). 
proved nor disproved; the best known upper bound on the length of a reset word is $\left(n^{3}-n\right) / 6$, as shown by Pin 8 .

While Eppstein [4] showed that the problem of deciding whether there exists a reset word of a given length $k$ is NP-complete, the complexity of deciding whether a shortest reset word has length $k$ is not known to be in NP. In his survey paper [12, Volkov asked for the precise complexity of this problem. In this paper, we show that deciding whether a shortest reset word has length $k$ is complete for the class DP, the closure of $\mathrm{NP} \cup$ coNP under finite intersections. In particular, since every DP-complete problem is both NP-hard and coNP-hard, it is unlikely that the problem of deciding the length of a shortest reset word lies in $\mathrm{NP} \cup \operatorname{coNP} 1$

The class DP is contained in the class $\mathrm{P}^{\mathrm{NP}}$, i.e. every problem in $\mathrm{DP}$ can be solved by a deterministic polynomial-time Turing machine that has access to an oracle for an NP-complete problem. In fact, two oracle queries suffice for this purpose. If one restricts the number of oracle queries to be logarithmic in the size of the input, one arrives at the class $\mathrm{P}^{\mathrm{NP}[\mathrm{log}]}$, which is believed to be a proper superclass of DP. We show that the problem of computing the length of a shortest reset word (as opposed to deciding whether it is equal to a given integer) is, in fact, complete for $\mathrm{FP}^{\mathrm{NP}[\log ]}$, the functional analogue of $\mathrm{P}^{\mathrm{NP}[\log ]}$. Hence, this problem seems to be even harder than deciding the length of a shortest reset word. Our result complements a recent result by Berlinkov [1], who showed that, unless $\mathrm{P}=\mathrm{NP}$, there is no polynomial-time algorithm that approximates the length of a shortest reset word within a constant factor.

For the more general problem of computing a shortest reset word (not only its length), we prove membership in $\mathrm{FP}^{\mathrm{NP}}$, the functional analogue of $\mathrm{P}^{\mathrm{NP}}$. While our lower bound of $\mathrm{FP}^{\mathrm{NP}[\mathrm{log}]}$ on computing the length of a shortest reset word carries over to this problem, we leave it as an open problem whether computing a shortest reset word is also $\mathrm{FP}^{\mathrm{NP}}$-hard.

Apart from studying problems related to computing a shortest reset word, we also consider the problem of computing a reset word of a given length (represented in unary). We observe that this problem is complete for the class FNP of search problems for which a solution can be verified in polynomial time. In other words: the problem is as hard as computing a satisfying assignment for a given Boolean formula.

\section{Preliminaries}

Let $\mathcal{A}=\langle Q, \Sigma, \delta\rangle$ be a deterministic finite automaton (DFA) with finite state set $Q$, finite alphabet $\Sigma$ and transition function $\delta: Q \times \Sigma \rightarrow Q$. The transitive closure of $\delta$ can be defined inductively by $\delta^{*}(q, \varepsilon)=q$ and $\delta^{*}(q, w a)=\delta\left(\delta^{*}(q, w), a\right)$ for each $q \in Q, w \in \Sigma^{*}$ and $a \in \Sigma$. We call any word $w \in \Sigma^{*}$ such that

\footnotetext{
${ }^{1}$ We have been informed that Gawrychowski [5] has shown DP-completeness of SHORTEST-RESET-WORD earlier, but his proof has never been published. While his reduction uses a five-letter alphabet, we prove hardness even over a binary alphabet.
} 
$\left|\left\{\delta^{*}(q, w) \mid q \in Q\right\}\right|=1$ a reset word for $\mathcal{A}$, and we say that $\mathcal{A}$ is synchronising if such a word exists. Note that, if $w$ is a reset word for $\mathcal{A}$, then so is $x w y$ for all $x, y \in \Sigma^{*}$.

We assume that the reader is familiar with basic concepts of complexity theory, in particular with the classes $\mathrm{P}, \mathrm{NP}$ and coNP. We will introduce the other classes that play a role in this paper on the fly. For details, see [7/10.

\section{Decision Problems}

The most fundamental decision problem concerning reset words is to decide whether a given deterministic finite automaton is synchronising. Černý [2] noted that it suffices to check for each pair $\left(q, q^{\prime}\right)$ of states whether there exists a word $w \in \Sigma^{*}$ with $\delta^{*}(q, w)=\delta^{*}\left(q^{\prime}, w\right)$. The latter property can obviously be decided in polynomial time. The best known algorithm for computing a reset word is due to Eppstein [4]: his algorithm runs in time $\mathrm{O}\left(|Q|^{3}+|Q|^{2} \cdot|\Sigma|\right)$. Computing a shortest reset word, however, cannot be done in polynomial time unless the following decision problems are in $\mathrm{P}$.

SHORT-RESET-WORD: Given a DFA $\mathcal{A}$ and a positive integer $k$, decide whether there exists a reset word for $\mathcal{A}$ of length $k$.

SHORTEST-RESET-WORD: Given a DFA $\mathcal{A}$ and a positive integer $k$, decide whether the minimum length of a reset word for $\mathcal{A}$ equals $k$.

If the parameter $k$ is given in unary, it is obvious that SHORT-RESET-WORD is in NP. However, even if $k$ is given in binary, this problem is in NP: since every synchronising automaton has a reset word of length $p(|Q|)$ (where $p$ is a low-degree polynomial, e.g. $\left.p(n)=\left(n^{3}-n\right) / 6\right)$, to establish whether there exists a reset word of length $k$, it suffices to guess a reset word of length $\min \{p(|Q|), k\}$. Eppstein 4 gave a matching lower bound by proving that SHORT-RESET-WORD is also NP-hard.

Regarding SHORTEST-RESET-WORD, Samotij [9] showed that the problem is NP-hard. We prove that SHORTEST-RESET-WORD is complete for DP, the class of all languages of the form $L=L_{1} \backslash L_{2}$ with $L_{1}, L_{2} \in \mathrm{NP}$. Since DP is a superclass of both NP and coNP, our result implies hardness for both of these classes. In fact, we show that SHORTEST-RESET-WORD is DP-hard even over a binary alphabet.

Theorem 1. SHORTEST-RESET-WORD is DP-complete.

Proof. It is easy to see that SHORTEST-RESET-WORD belongs to DP: indeed, we can write SHORTEST-RESET-WORD as the difference of SHORT-RESET-WORD and SHORT-RESET-WORD ${ }^{-}$, where

$$
\text { SHORT-RESET-WORD }^{-}=\{(\mathcal{A}, k+1) \mid(\mathcal{A}, k) \in \text { SHORT-RESET-WORD }\},
$$

a problem which is obviously in NP (even if $k$ is given in binary). 
It remains to prove that SHORTEST-RESET-WORD is DP-hard. We reduce from the canonical DP-complete problem SAT-UNSAT: given two Boolean formulae $\varphi$ and $\psi$ (in $\mathrm{CNF}$ ), decide whether $\varphi$ is satisfiable and $\psi$ is unsatisfiable. More precisely, we show how to construct (in polynomial time) from a pair $(\varphi, \psi)$ of Boolean formulae in CNF over propositional variables $X_{1}, \ldots, X_{k}$ a synchronising automaton $\mathcal{A}$ over the alphabet $\Sigma=\{0,1\}$ with the following properties:

1. If $\varphi$ and $\psi$ are satisfiable, then there exists a reset word of length $k+2$.

2. If $\varphi$ is satisfiable and $\psi$ is unsatisfiable, then a shortest reset word has length $k+3$.

3. If $\varphi$ is unsatisfiable, then every reset word has length at least $k+4$.

From 1.-3. we get that $\varphi$ is satisfiable and $\psi$ is unsatisfiable if and only if a shortest reset word has length $k+3$.

Given formulae $\varphi=C_{1} \wedge \ldots \wedge C_{n}$ and $\psi=D_{1} \wedge \ldots \wedge D_{n}$ where, without loss of generality, $\varphi$ and $\psi$ have the same number $n$ of clauses, and no propositional variable occurs in both $\varphi$ and $\psi$, the automaton $\mathcal{A}$ consists of the states $s, t_{1}$, $t_{2}, p_{i, j}$ and $q_{i, j}, i \in\{1, \ldots, n\}, j \in\{\perp, \top, 1, \ldots, k\}$; the transitions are depicted in Fig. 1 an edge from $p$ to $q$ labelled with $\Sigma^{\prime} \subseteq \Sigma$ has the meaning that $\delta(p, a)=q$ for each $a \in \Sigma^{\prime}$. The sets $\Sigma_{i}^{j} \subseteq \Sigma$ are defined by $0 \in \Sigma_{i}^{j} \Leftrightarrow \neg X_{j} \in C_{i}$ and $1 \in \Sigma_{i}^{j} \Leftrightarrow X_{j} \in C_{i}$, and the sets $\Gamma_{i}^{j} \subseteq \Sigma$ are defined by $0 \in \Gamma_{i}^{j} \Leftrightarrow \neg X_{j} \in D_{i}$ and $1 \in \Gamma_{i}^{j} \Leftrightarrow X_{j} \in D_{i}$. Hence, e.g. $0 \in \Sigma_{i}^{j}$ if we can satisfy the $i$ th clause of $\varphi$ by setting variable $X_{j}$ to false.

Clearly, $\mathcal{A}$ can be constructed in polynomial time from $\varphi$ and $\psi$. To establish our reduction, it remains to verify $1 .-3$.

To prove 1., assume that $\varphi$ and $\psi$ are both satisfiable. Since $\varphi$ and $\psi$ share no variable, there exists an assignment $\alpha:\left\{X_{1}, \ldots, X_{k}\right\} \rightarrow\{$ true, false $\}$ that satisfies both $\varphi$ and $\psi$. We claim that the word $01 w$, where $w=w_{1} \ldots w_{k} \in$ $\{0,1\}^{k}$ is defined by $w_{j}=1 \Leftrightarrow \alpha\left(X_{j}\right)=$ true, resets $\mathcal{A}$ to $s$. Clearly, $\delta^{*}(q, w)=s$ for all states $q$ that are not of the form $q=p_{i, \perp}, q=p_{i, \top}, q=q_{i, \perp}$ or $q=$ $q_{i, \top}$. Since $\delta^{*}\left(p_{i, \perp}, 01\right)=\delta^{*}\left(p_{i, \top}, 01\right)=p_{i, 1}$ and $\delta^{*}\left(q_{i, \perp}, 01\right)=\delta^{*}\left(q_{i, \top}, 01\right)=$ $q_{i, 1}$ for each $i=1, \ldots, n$, it suffices to show that $\delta^{*}\left(p_{i, 1}, w\right)=\delta^{*}\left(q_{i, 1}, w\right)=s$ for all $i$. To prove that $\delta^{*}\left(p_{i, 1}, w\right)=s$, consider the least $j$ such that either $X_{j} \in C_{i}$ and $\alpha\left(X_{j}\right)=$ true or $\neg X_{j} \in C_{i}$ and $\alpha\left(X_{j}\right)=$ false (such $j$ exists since $\alpha$ satisfies $\varphi)$. We have $\delta^{*}\left(p_{i, 1}, w_{1} \ldots w_{j-1}\right)=p_{i, j}$ and $\delta\left(p_{i, j}, w_{j}\right)=s$ and therefore also $\delta^{*}\left(p_{i, 1}, w\right)=s$. The argument for $\delta^{*}\left(q_{i, 1}, w\right)=s$ is analogous.

Towards proving 2., assume that $\varphi$ is satisfiable but $\psi$ is not. Consider an assignment $\alpha:\left\{X_{1}, \ldots, X_{k}\right\} \rightarrow\{$ true, false $\}$ that satisfies $\varphi$. It follows with the same reasoning as above that the word $01 w 1$, where $w \in\{0,1\}^{k}$ is defined by $w_{j}=1 \Leftrightarrow \alpha\left(X_{j}\right)=$ true, resets $\mathcal{A}$ to $s$.

To show that a shortest reset word has length $k+3$, it remains to show that there exists no reset word of length $k+2$. Towards a contradiction, assume that $w=w_{1} \ldots w_{k+2}$ is such a word. Note that $w$ resets $\mathcal{A}$ to $s$ and that there exists $l \geq 2$ such that $\delta^{*}\left(q_{i, \perp}, w_{1} \ldots w_{l}\right)=q_{i, 1}$ and $\delta^{*}\left(q_{i, 1}, w_{l+1} \ldots w_{k+2}\right)=s$ for all $i=1, \ldots, n$. Define $\alpha:\left\{X_{1}, \ldots, X_{k}\right\} \rightarrow\{$ true, false $\}$ by setting $\alpha\left(X_{j}\right)=$ true $\Leftrightarrow w_{l+j}=1$. Since $l \geq 2$ but $\delta^{*}\left(q_{i, 1}, w_{l+1} \ldots w_{k+2}\right)=s$, for each $i$ there 


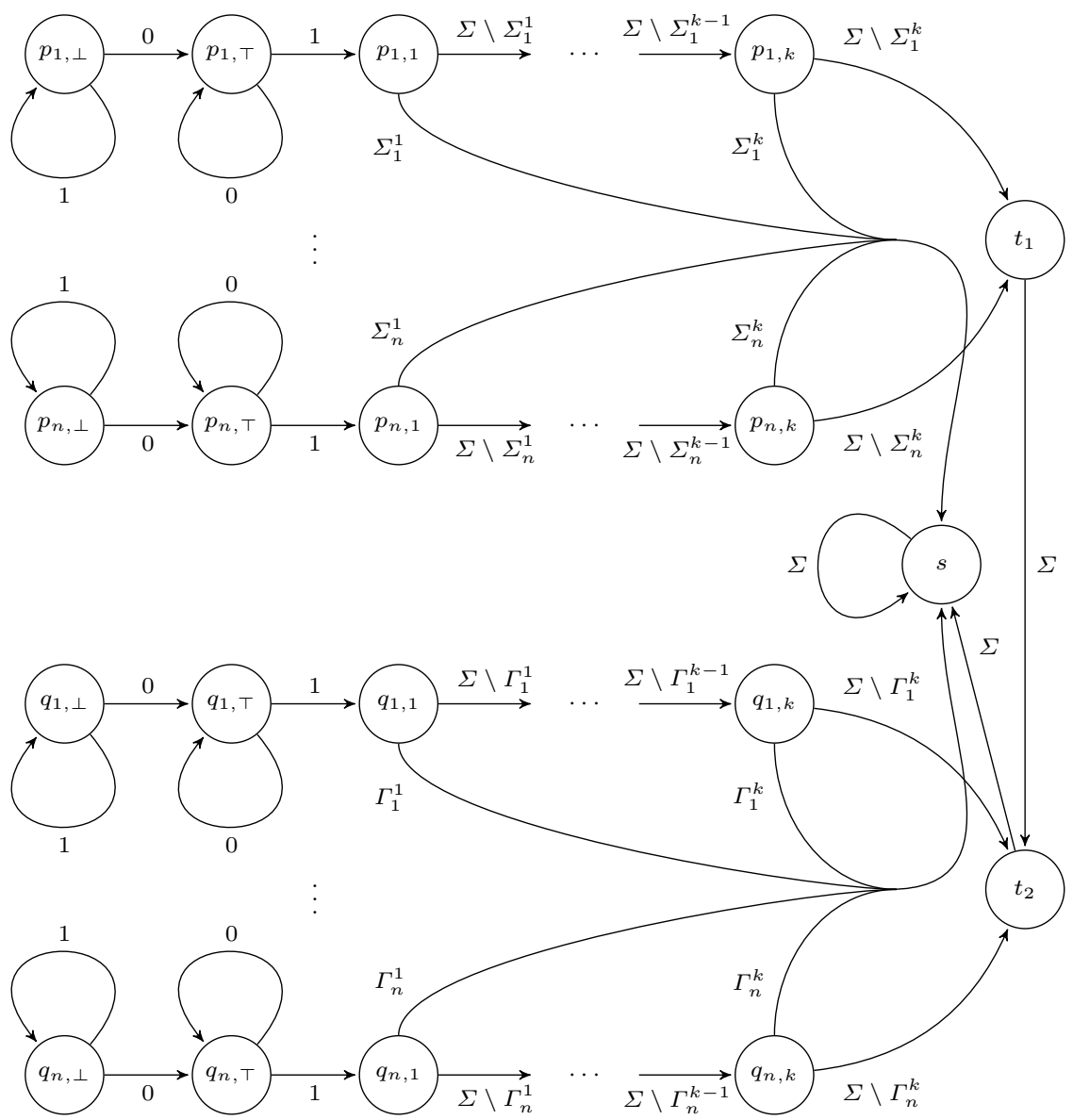

Fig. 1. Reducing SAT-UnSAT to ShORTEST-RESET-WORD

must exist $j \in\{1, \ldots, k\}$ such that $\delta\left(q_{i, j}, w_{l+j}\right)=s$. But then either $X_{j} \in D_{i}$ and $\alpha\left(X_{j}\right)=$ true or $\neg X_{j} \in D_{i}$ and $\alpha\left(X_{j}\right)=$ false. Hence, $\alpha$ is a satisfying assignment for $\psi$, contradicting our assumption that $\psi$ is unsatisfiable.

Finally, assume that $\varphi$ is unsatisfiable. With the same reasoning as in the previous case, it follows that there is no reset word of length $k+3$.

The above reduction shows DP-hardness for an alphabet size of $|\Sigma|=2$. For the special case of only one input letter, note that each reset word is of the form $1^{n}$ for some $n$. Asking whether there exists a reset word of length $k$ thus collapses to the question whether $1^{k}$ is a reset word for $\mathcal{A}$. This property can be decided with logarithmic space. Hence, both problems, SHORT-RESET-WORD and SHORTEST-RESET-WORD, are in LOGSPACE for $|\Sigma|=1$. 


\section{Search problems}

In this section, we leave the realm of decision problems and enter the (rougher) territory of search problems, where the task is not only to decide whether a reset word of some length exists, but to compute a suitable word (or its length). More precisely, we deal with the following search problems:

- Given a DFA $\mathcal{A}$ and a positive integer $k$ in unary, compute a reset word for $\mathcal{A}$ of length $k$.

- Given a DFA $\mathcal{A}$, compute the length of a shortest reset word for $\mathcal{A}$.

- Given a DFA $\mathcal{A}$, compute a shortest reset word for $\mathcal{A}$.

Let us start with the first problem of computing a reset word of a given length. It turns out that this problem is complete for the class FNP of search problems where the underlying binary relation is both polynomially balanced and decidable in polynomial time.

Proposition 2. The problem of computing a reset word of a given length is FNP-complete.

Proof. Membership in FNP follows from the fact that the binary relation

$$
\left\{\left(\left(\mathcal{A}, 1^{k}\right), w\right) \mid w \text { is a reset word for } \mathcal{A} \text { of length } k\right\}
$$

is polynomially balanced and polynomial-time decidable.

To prove hardness, we reduce from FSAT, the problem of computing a satisfying assignment for a given Boolean formula in conjunctive normal form. To this end, we describe two polynomial-time computable functions $f$ and $g$, where $f$ computes from a CNF formula $\varphi$ a synchronising automaton $\mathcal{A}=f(\varphi)$ over the alphabet $\{0,1\}$ and a unary number $k \in \mathbb{N}$, and $g$ computes from $\varphi$ and $w \in \Sigma^{*}$ an assignment for $\varphi$, such that, if $w$ is a reset word for $\mathcal{A}$ of length $k$, then the generated assignment satisfies $\varphi$.

Eppstein 4 showed how to compute in polynomial time, given a CNF formula $\varphi=C_{1} \wedge \ldots \wedge C_{n}$ over the variables $X_{1}, \ldots, X_{k}$, an automaton $\mathcal{A}_{\varphi}$ over the alphabet $\{0,1\}$ with the following two properties:

1. A word $w=w_{1} \cdots w_{k}$ is a reset word for $\mathcal{A}$ if and only if the assignment $\alpha$, defined by $\alpha\left(X_{j}\right)=$ true $\Leftrightarrow w_{j}=1$, satisfies $\varphi$.

2. An assignment $\alpha:\left\{X_{1}, \ldots, X_{k}\right\} \rightarrow\{$ true, false $\}$ satisfies $\varphi$ if and only if the word $w \in\{0,1\}^{k}$, defined by $w_{j}=1 \Leftrightarrow \alpha\left(X_{j}\right)=$ true, is a reset word for $\mathcal{A}$.

(Note that the reduction we use to prove Theorem 1 has similar properties and could also be used.)

Hence, we can choose $f$ to be the function that maps $\varphi$ to $\left(\mathcal{A}_{\varphi}, 1^{k}\right)$ and $g$ to be the function that maps $(\varphi, w)$ to the corresponding assignment $\alpha$. (If $|w| \neq k$, then $\alpha$ can be chosen arbitrarily.) 


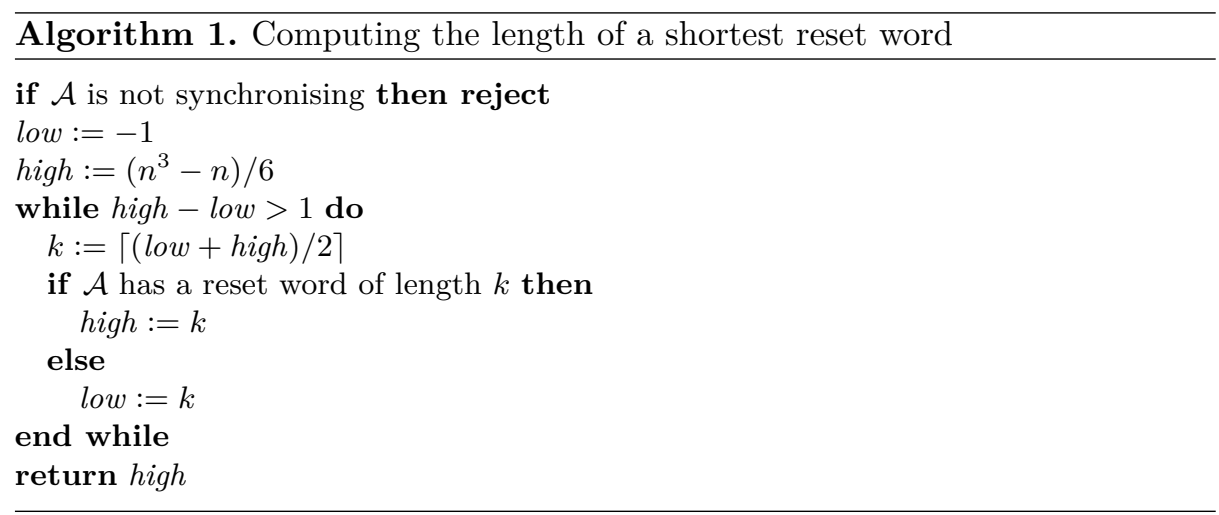

Remark 3. Note that the mapping $f:\{0,1\}^{k} \rightarrow\{\text { true, false }\}^{\left\{X_{1}, \ldots, X_{k}\right\}}$, defined by $f(w)\left(X_{j}\right)=$ true $\Leftrightarrow w_{j}=1$, is a bijection. Eppstein's reduction shows that one can compute from a Boolean formula $\varphi$ over the variables $\left\{X_{1}, \ldots, X_{k}\right\}$ an automaton $\mathcal{A}$ such that $f$ remains a bijection when one restricts the domain to reset words for $\mathcal{A}$ and the range to assignments that satisfy $\varphi$. Therefore, his reduction can be viewed as a parsimonious reduction from \#SAT, the problem of counting all satisfying assignments of a given Boolean formula, to the problem of counting all reset words of a given length (represented in unary). Since the first problem is complete for \#P [11], the second problem is \#P-hard. On the other hand, it is easy to see that the second problem is in \#P. Hence, this problem is \#P-complete.

Next, we consider the problem of computing the length of a shortest reset word for a given automaton: we establish that this problem is complete for the class $\mathrm{FP}^{\mathrm{NP}[\log ]}$ of all problems that are solvable by a polynomial-time algorithm with access to an oracle for a problem in NP where the number of queries is restricted to $\mathrm{O}(\log n)$.

Theorem 4. The problem of computing the length of a shortest reset word is $\mathrm{FP}^{\mathrm{NP}[\log ]}$-complete.

Proof. To prove membership in $\mathrm{FP}^{\mathrm{NP}[\log ]}$, consider Algorithm 1 which is a binary-search algorithm for determining the length of a shortest reset word for an automaton $\mathcal{A}$ with $n$ states. The algorithm is executed in polynomial time: the while loop is repeated $\mathrm{O}(\log n)$ times and asks $\mathrm{O}(\log n)$ queries to the oracle, which is used for determining whether $\mathcal{A}$ has a reset word of a given length.

Krentel [6] showed that MAX-SAT-SIZE, the problem of computing the maximum number of simultaneously satisfiable clauses of a CNF formula, is complete for $\mathrm{FP}^{\mathrm{NP}[\log ]}$. Therefore, to establish $\mathrm{FP}^{\mathrm{NP}[\log ]}$-hardness, it suffices to give a reduction from MAX-SAT-SIZE to our problem. Such a reduction consists of two polynomial-time computable functions $f$ and $g$ with the following properties: $f$ computes from a CNF formula $\varphi$ a (synchronising) automaton $\mathcal{A}=f(\varphi)$, and $g$ computes from $\varphi$ and $l \in \mathbb{N}$ a new number $g(\varphi, l) \in \mathbb{N}$ such that, if $l$ is the 
length of a shortest reset word for $\mathcal{A}$, then the maximum number of simultaneously satisfiable clauses in $\varphi$ equals $g(\varphi, k)$.

Given a formula $\varphi=C_{1} \wedge \ldots \wedge C_{n}$ over propositional variables $X_{1}, \ldots, X_{k}$, the resulting automaton $\mathcal{A}$ is depicted in Fig. 2 The input alphabet is $\Sigma:=$ $\{0,1, \$\}$, and the sets $\Sigma_{i}^{j} \subseteq \Sigma$ are defined as in the proof of Theorem 1 , we set $\lambda:=k+n(n+4)$. The behaviour of the transition function on vertices of the form $r_{i, j}$ is defined as follows:

$-\delta\left(r_{i, j}, \$\right)=p_{i, 1}$ for all $j \in\{-2, \ldots, n+1\}$;

$-\delta\left(r_{i, j}, 1\right)=r_{i, j+1}, \delta\left(r_{i, j}, 0\right)=r_{i,-2}$ for all $j \in\{-2,-1, i\}$;

$-\delta\left(r_{i, j}, 1\right)=r_{i,-2}, \delta\left(r_{i, j}, 0\right)=r_{i, j+1}$ for all $j \in\{0, \ldots, i-1, i+1, \ldots, n\}$;

$-\delta\left(r_{i, n+1}, 1\right)=r_{i,-2}, \delta\left(r_{i, n+1}, 0\right)=s$.

It is not difficult to see that $\mathcal{A}$ can be constructed in polynomial time from $\varphi$. Moreover, we claim that, for each $m \in\{0,1, \ldots, n\}$, there exists an assignment that satisfies at least $n-m$ clauses of $\varphi$ if and only if $\mathcal{A}$ has a reset word of length $1+\lambda+k+m(n+4)$. Hence, if $l$ is the length of a shortest reset word for $\mathcal{A}$, then the maximal number of simultaneously satisfiable clauses of $\varphi$ is given by $n-\left[\frac{\max \{0, l-1-\lambda-k\}}{n+4}\right]$. Clearly, this number can be computed in polynomial time from $\varphi$ and $l$.

$(\Rightarrow)$ Assume that $\alpha:\left\{X_{1}, \ldots, X_{k}\right\} \rightarrow\{$ true, false $\}$ is an assignment that satisfies all clauses except (possibly) the clauses $C_{i_{1}}, \ldots, C_{i_{m}}$, and consider the word

$$
w:=\$ 1^{\lambda} x_{1} \ldots x_{k} z_{i_{1}} \ldots z_{i_{m}},
$$

where $z_{i}=110^{i} 10^{n-i+1} \in\{0,1\}^{n+4}$ for $i \in\{1, \ldots, n\}$ and

$$
x_{j}:= \begin{cases}1 & \text { if } \alpha\left(X_{j}\right)=\text { true } \\ 0 & \text { otherwise }\end{cases}
$$

Note that $w$ has length $1+\lambda+k+m(n+4)$. We claim that $w$ resets $\mathcal{A}$ to $s$. Since reading $\$$ has the effect of going from each state of the form $p_{i, j}, q_{i, j}$ or $r_{i, j}$ to $p_{i, 1}$ and from $t$ to $s$, and reading $1^{\lambda}$ has the effect of going from $p_{i, 1}$ to $q_{i, 1}$, it suffices to show that $\delta^{*}\left(q_{i, 1}, x_{1} \ldots x_{k} z_{i_{1}} \ldots z_{i_{m}}\right)=s$. If $C_{i}$ is satisfied by $\alpha$, then this follows from the fact that there exists $j$ such that $\delta\left(q_{i, j}, x_{j}\right)=s$. Otherwise, we have $\delta^{*}\left(q_{i, 1}, x_{1} \ldots x_{k}\right)=r_{i,-2}, \delta^{*}\left(r_{i,-2}, z_{j}\right)=r_{i,-2}$ for all $j \neq i$, but $\delta^{*}\left(r_{i,-2}, z_{i}\right)=s$. Since $i \in\left\{i_{1}, \ldots, i_{m}\right\}$, this implies that $\delta^{*}\left(q_{i, 1}, x_{1} \ldots x_{k} z_{i_{1}} \ldots z_{i_{m}}\right)=s$.

$(\Leftarrow)$ Assume that $\mathcal{A}$ has a reset word of length $1+\lambda+k+m(n+4)$, and let $w$ be a shortest reset word for $\mathcal{A}$. We claim that $w$ has the form $w=\$ u$ or $w=u \$$ for $u \in\{0,1\}^{*}$. Otherwise, $w=u \$ v$ for $u, v \in \Sigma^{+}$. Towards a contradiction, we distinguish the following two cases: $|u| \leq \lambda$ and $|u|>\lambda$. If $|u| \leq \lambda$, then $\delta^{*}\left(p_{i, 1}, u \$\right)=p_{i, 1}$ for all $i=1, \ldots, n$, and the word $\$ v$ would be a shorter reset word than $w$. Now assume that $|u|>\lambda$. It must be the case that $\delta^{*}\left(p_{i, 1}, u\right) \neq s$ for some $i \in\{1, \ldots, n\}$ because otherwise $\$ u$ would be a shorter reset word than $w$. But then $\delta^{*}\left(p_{i, 1}, u \$\right)=p_{i, 1}$. Hence, since $w$ resets $\mathcal{A}$ 


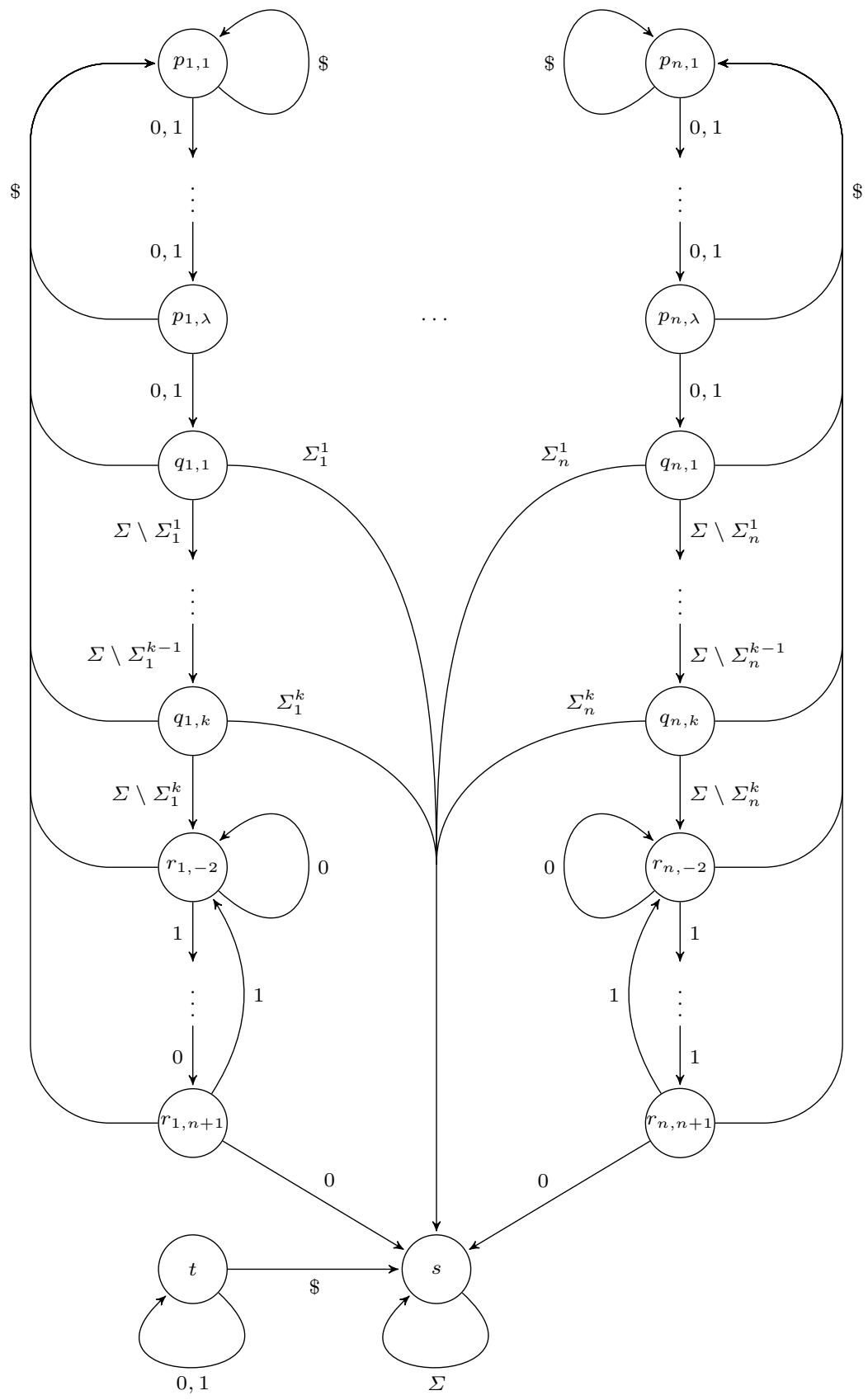

Fig. 2. Reducing MAX-SAT-SIZE to computing the length of a shortest reset word 
to $s$ and the shortest path from $p_{i, 1}$ to $s$ has length greater than $\lambda,|v|>\lambda$ and $|w|>1+2 \lambda \geq 1+\lambda+k+n(n+4) \geq 1+\lambda+k+m(n+4)$, a contradiction.

Now, if $\varphi$ is satisfiable, we are done. Otherwise, let us fix $u \in\{0,1\}^{*}$ such that $w=\$ u$ or $w=u \$$. Since $\varphi$ is not satisfiable, $|u| \geq \lambda+k$. Let $u=y x_{1} \ldots x_{k} z$ where $y, z \in\{0,1\}^{*},|y|=\lambda$, and $x_{j} \in\{0,1\}$ for all $j=1, \ldots, k$. Now consider the assignment $\alpha$ defined by

$$
\alpha\left(X_{j}\right)= \begin{cases}\text { true } & \text { if } x_{j}=1 \\ \text { false } & \text { otherwise }\end{cases}
$$

Moreover, let

$$
I:=\left\{i \in\{1, \ldots, n\} \mid C_{i} \text { is not satisfied by } \alpha\right\} .
$$

We claim that $|I| \leq m$ (so $\alpha$ satisfies at least $n-m$ clauses of $\varphi$ ). To see this, first note that $\delta^{*}\left(p_{i, 1}, y x_{1} \ldots x_{k}\right)=r_{i,-2}$ for all $i \in I$. Hence, we must have that $\delta^{*}\left(r_{i,-2}, z\right)=s$ for all such $i$. By the construction of $\mathcal{A}$, this is only possible if $z$ contains the word $110^{i} 10^{n-i+1}$ as an infix for each $i \in I$. Since these infixes cannot overlap, $|z| \geq|I| \cdot(n+4)$. On the other hand, since $|u| \leq \lambda+k+m(n+4)$, we must have $|z| \leq m(n+4)$. Hence, $|I| \leq m$.

The construction we have presented to prove Theorem 4 uses a three-letter alphabet. With a little more effort, we can actually reduce the alphabet to an alphabet with two letters 0 and 1: For each state $q \notin\{s, t\}$ of $\mathcal{A}$, there are three states $(q, 0),(q, 1)$ and $(q, 2)$ in the new automaton $\mathcal{A}^{\prime}$. Additionally, $\mathcal{A}^{\prime}$ contains the states $(t, 0),(t, 1)$ and $s$. The new transition function $\delta^{\prime}$ is defined as follows:

$$
\begin{array}{ll}
\delta^{\prime}((q, 0), 0)=(q, 1), & \delta^{\prime}((q, 0), 1)=(q, 2), \\
\delta^{\prime}((q, 1), 0)=(q, 1), & \delta^{\prime}((q, 1), 1)=(\delta(q, \$), 2), \\
\delta^{\prime}((q, 2), 0)=(\delta(q, 0), 0), & \delta^{\prime}((q, 2), 1)=(\delta(q, 1), 0)
\end{array}
$$

for all $q \notin\{s, t\}$, and

$$
\begin{aligned}
& \delta^{\prime}((t, 0), 0)=s, \\
& \delta^{\prime}((t, 0), 1)=(t, 1), \\
& \delta^{\prime}((t, 1), 0)=(t, 0), \\
& \delta^{\prime}(s, 0)=s, \\
& \delta^{\prime}((t, 1), 1)=(t, 1), \\
& \delta^{\prime}(s, 1)=s .
\end{aligned}
$$

Intuitively, taking a transition in $\mathcal{A}$ corresponds to taking two transitions in $\mathcal{A}^{\prime}$. It is not difficult to see that a shortest reset word for $\mathcal{A}^{\prime}$ has length $2 l$ if a shortest reset word for $\mathcal{A}$ has length $l$.

For the potentially harder problem of computing a shortest reset word (not only its length), we can only prove membership in $\mathrm{FP}^{\mathrm{NP}}$, the class of all search problems that are solvable in polynomial time using an oracle for a problem in NP (without any restriction on the number of queries). Of course, hardness for $\mathrm{FP}^{\mathrm{NP}[\log ]}$ carries over from our previous result. We have not been able to close the gap between the two bounds. To the best of our knowledge, the same situation occurs e.g. for MAX-SAT, where the aim is to find an assignment of a given Boolean formula that satisfies as many clauses as possible. 


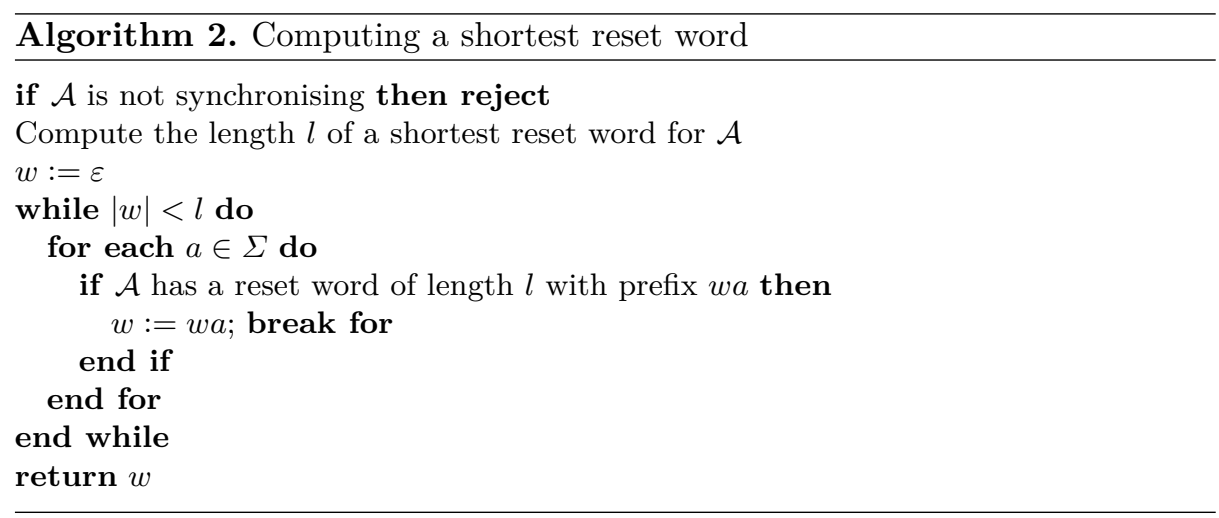

Theorem 5. The problem of computing a shortest reset word is in $\mathrm{FP}^{\mathrm{NP}}$ and hard for $\mathrm{FP}^{\mathrm{NP}}[\mathrm{log}]$.

Proof. To prove membership in $\mathrm{FP}^{\mathrm{NP}}$, consider Algorithm 2 for computing a shortest reset word for an automaton $\mathcal{A}$ over any finite alphabet $\Sigma$. The algorithm obviously computes a reset word of length $l$, which is the length of a shortest reset word. To see that the algorithm runs in polynomial time if it has access to an NP oracle, note that deciding whether $\mathcal{A}$ has a reset word of a given length with a given prefix is in NP (since a nondeterministic polynomial-time algorithm can guess such a word). Moreover, as we have shown above, computing the length of a shortest reset word can be done by a polynomial-time algorithm with access to an NP oracle.

Hardness for $\mathrm{FP}^{\mathrm{NP}[\mathrm{log}]}$ follows from Theorem 4 since the problem of computing the length of a shortest reset word is trivially reducible to the problem of computing a shortest reset word: an instance of the former problem is also an instance of the latter problem, and a solution of the latter problem can be turned into a solution of the former problem by computing its length.

\section{Conclusion}

We have investigated several decision problems and search problems about finding reset words in finite automata. The results we have obtained shed more light on the difficulty of computing such words. In particular, deciding whether for a given automaton a shortest reset word has length $k$ is DP-complete, and computing the length of a shortest reset word is $\mathrm{FP}^{\mathrm{NP}[\log ]}$-complete, i.e. as hard as calculating the maximum number of simultaneously satisfiable clauses of a Boolean formula. A summary of all our results is depicted in Fig. 3. (See [7]10] for the relationships between the referred complexity classes.)

Acknowledgements. We thank an anonymous reviewer for pointing out [5]. Moreover, we are grateful to Christof Löding and Wolfgang Thomas for helpful comments on an early draft of this paper. 

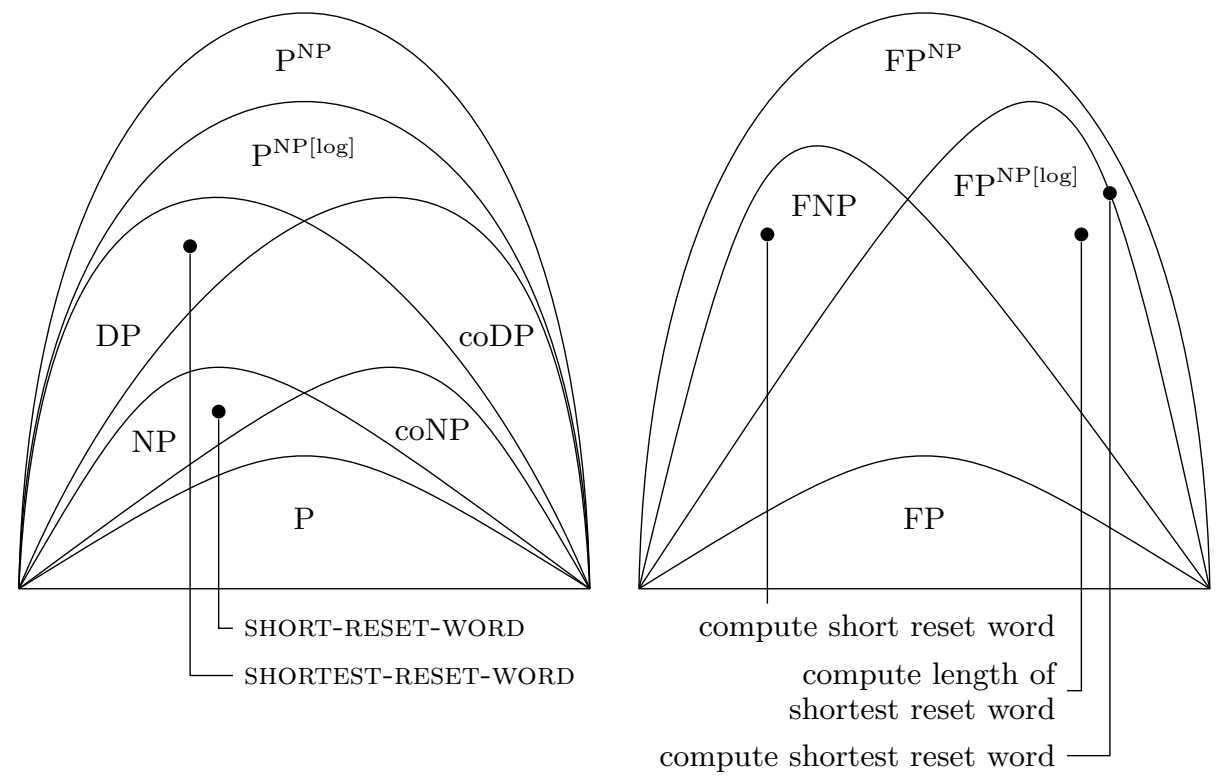

Fig. 3. Summary of results

\section{References}

1. M. V. Berlinkov. Approximating the length of synchronizing words. In Proc. CSR 2010, LNCS 6072. Springer, 2010.

2. J. Černý. Poznámka k. homogénnym experimentom s konecnými automatmi. Matematicko-fyzikalny Časopis Slovensk. Akad. Vied, 14(3):208-216, 1964.

3. J. Černý, A. Pirická, and B. Rosenauerová. On directable automata. Kybernetica, $7(4): 289-298,1971$.

4. D. Eppstein. Reset sequences for monotonic automata. SIAM Journal on Computing, 19(3):500-510, 1990.

5. P. Gawrychowski. Complexity of shortest synchronizing word. Unpublished manuscript, April 2008.

6. M. W. Krentel. The complexity of optimization problems. Journal of Computer and System Sciences, 36:490-509, 1988.

7. C. H. Papadimitriou. Computational complexity. Addison-Wesley, 1994.

8. J.-É. Pin. On two combinatorial problems arising from automata theory. Annals of Discrete Mathematics, 17:535-548, 1983.

9. W. Samotij. A note on the complexity of the problem of finding shortest synchronizing words. In Proc. AutoMathA 200\%. University of Palermo (CD), 2007.

10. A. L. Selman. A taxonomy of complexity classes of functions. Journal of Computer and System Sciences, 48(2):357-381, 1994.

11. L. G. Valiant. The complexity of computing the permanent. Theoretical Computer Science, 8:189-201, 1979.

12. M. V. Volkov. Synchronizing automata and the Černý conjecture. In Proc. LATA 2008, LNCS 5196, pages 11-27. Springer, 2008. 